\title{
ACIDENTES POR MORDEDURAS DE CÃES E GATOS NO MUNICÍPIO DE PINHAIS, BRASIL DE 2002 A 2005
}

\author{
(Dog and cat bite accidents in Pinhais, Brazil, from 2002 to 2005)
}

\author{
FORTES, F. S. ${ }^{1}$; WOUK, A.F.P.F ${ }^{1}$; BIONDO, A. W. ${ }^{1.2}$; BARROS, C. C. ${ }^{3}$
}

1. Departamento de Medicina Veterinária, Universidade Federal do Paraná. 2. Department of Pathobiology, College of Veterinary Medicine, Urbana-Champaign. 3. Centro de Controle de Zoonoses, Vigilância Sanitária Municipal, Rua Alto Paraná.

\begin{abstract}
RESUMO - O presente estudo teve por objetivo avaliar as características dos acidentes humanos de mordeduras causados por cães e gatos no município de Pinhais, Paraná, Brasil no período de 2002 a 2005. Foram avaliados os dados referentes a atendimentos anti-rábicos suspeitos das Unidades de Saúde Municipais de 2.163 fichas do Sistema de Informação de Agravos de Notificação (SINAN). As agressões ocorreram, predominantemente, em pacientes com idade superior a doze anos $(61,4 \%)$, do sexo masculino $(57,3 \%)$, com ferimentos úni$\cos (58,3 \%)$ e superficiais $(49,7 \%)$ localizados nos membros inferiores $(33,3 \%)$. A mordedura $(81,9 \%)$ foi o tipo de exposição mais freqüente, sendo causada principalmente por cães $(95,9 \%) ; 51,0 \%$ dos animais foram considerados sadios no momento do acidente. A agressão foi provocada pela vítima em 508 casos $(23,5 \%)$ e em sua maioria durante horas de lazer $(47,8 \%)$. Março, abril e maio foram os meses de menor ocorrência. O formulário do SINAN não fornece informações sobre a situação de domicílio do animal, seu relacionamento com a vítima e o local onde a agressão ocorreu, dados de grande importância para indicar o perfil do animal agressor e da vítima. Em conclusão, o contínuo monitoramento de acidentes por mordeduras pode ser um parâmetro da efetividade nas atividades de educação em saúde. Além disso, pode ser utilizado como base nos programas de guarda responsável, com foco na prevenção de acidentes por mordeduras.
\end{abstract}

\begin{abstract}
The present study aimed to evaluate the characteristics of bite accidents in human beings caused by dogs and cats in the city of Pinhais, Paraná, Brazil from 2002 to 2005. Data of suspicious anti-rabies attendance in City Health Units from a total of 2,163 forms from Brazilian National Disease Notification System (BNDNS) were evaluated. Aggression mainly occurred in patients older than 12 years $(61.4 \%)$, males $(57.3 \%)$, with single $(58.3 \%)$ and superficial $(49.7 \%$ ) wounds, mostly in rear limbs $(33.3 \%)$. Bite was referred as the most frequent exposure $(81.9 \%)$, mainly by dogs $(95.9 \%)$; a total of $51.0 \%$ of animals were considered healthy at the moment of the accident. Aggression was provoked by the victim in 508 cases $(23.5 \%)$ and mostly during vacation hours (47.8\%). March, April and May were the months with the lowest frequency. As previously referred, BNDNS form does not supply information on animal housing status, relationship with the victim and local of aggression, important data to indicate the aggressive animal and victim profiles. In conclusion, continuous monitoring of bite accidents may be used as a parameter of effectiveness of health education activities. Moreover, it may be used as basis for responsible guard programs, with focus in the bite accident prevention.
\end{abstract}

Key-words: bite wounds; dogs; responsible guard.

Palavras-chave: mordedura; cães; guarda responsável. 
Acidentes por mordeduras de cães e gatos no município de Pinhais, Brasil de 2002 a 2005

\section{INTRODUÇÃO}

A convivência com animais de estimação traz inúmeros benefícios psicológicos, fisiológicos e sociais aos seres humanos, porém, a criação inadequada dada a muitos animais aumenta o risco de agressão às pessoas, além de possibilitar a transmissão de doenças (MUNDIM et al., 2007).

Dentre os fatores que contribuem para o aumento da agressividade animal destacam-se o número elevado de animais mantidos em residências particulares, a falta de higiene no lugar onde vivem, os maus tratos a estes animais, o livre acesso dos mesmos às ruas e residências vizinhas, e a permanência dos animais em locais que dificultam sua movimentação natural (SCHOENDORFER, 2001). A agressão pode ser causada ainda por determinados estímulos como dor, dominância, alteração hormonal, medo, proteção a alimentos, pessoas, filhotes, território ou predatória (MUNDIM et al., 2007). O comportamento agressivo, assim como qualquer outra forma de conduta animal, pode ser ensinados ou causados propositalmente (ETTINGER e FELDMAN, 1997). Isto porque a agressividade é expressão da interação entre múltiplos fatores biológicos, psicológicos, sociais e ambientais, tais como as condições dos animais, do ambiente em que vivem, das interações estabelecidas e dos vínculos criados, especialmente com os seres humanos (BEAVER, 2001).

As mordeduras causadas por cães são objeto de grande preocupação devido a possibilidade de transmissão de zoonoses, como a raiva, de desenvolvimento de infecções secundárias, de seqüelas físicas e psicológicas, entre outras (PLAUT et al., 1996). Estes acidentes constituem portanto grave problema para a comunidade, para outros animais e para a saúde pública (MUNDIM, 2005; SCHABBACH, 2004). Além disso, os custos econômicos e sociais direcionados ao tratamento médico dos acidentados são elevados, consumindo recursos que poderiam ser investidos em programas de promoção à saúde que beneficiariam um grande número de pessoas (CHANG et al., 1997; OVERALL e LOVE, 2001). Os acidentes humanos causados por animais, principalmente os cães, ocorrem numa freqüência bastante elevada no Brasil (DEL CIAMPO et al., 2000).

Estudos mostram que as crianças são consideradas de alto risco para ataque de cães, e a mordedura é influenciada pela raça, comportamento e proprietário dos animais, pelas crianças e seus pais e que, portanto, a estratégia de prevenção deve ser focada na educação da população e no treino dos cães e de seus proprietários (TAN et al., 2004; SCHALAMON et al., 2006).

A raiva é uma zoonose letal causada por vírus da família Rhabdoviridae, gênero Lyssavirus, que pode acometer todos os mamíferos (HUGH-JONES et al., 1995) e constitui um problema sério de saúde pública nas regiões norte e nordeste do Brasil (ARAÚJO, 2001). As autoridades de saúde pública têm implantado medidas para o controle da raiva no Brasil, determinando grande redução dos casos de raiva humana e animal. Entretanto, não há redução proporcional no número de tratamentos anti-rábicos pós-exposição em pacientes vítimas de agressões animais (MINISTÉRIO DA SAÚDE, 2006).

O Paraná é considerado área controlada para raiva e a doença limitada aos municípios de fronteira com o Paraguai, não tendo sido registrado um único caso de raiva humana ou canina nos últimos vinte anos em Curitiba e região metropolitana (MINISTÉRIO DA SAÚDE, 2002). No entanto, a região de Curitiba não pode ser considerada erradicada para a raiva, uma vez que foram registrados vários casos em morcegos não hematófagos (frugívoros, insetívoros, etc) neste período, mostrando o risco potencial de reintrodução da doença através de animais silvestres (FENNER et al., 1993, ARAÚJO, 2001, ACHA e SZYFRES, 2003).

O objetivo do presente trabalho foi avaliar as características dos acidentes humanos de mordeduras causados por cães e gatos notificados nas unidades básicas de saúde situadas no município de Pinhais, região metropolitana de Curitiba, Brasil, no período de 2002 a 2005, com o intuito de obter subsídios para a prevenção de mordeduras no município, e conseqüente redução do risco de transmissão da raiva.

\section{MATERIAIS E MÉTODOS}

O presente estudo teve por base a análise retrospectiva dos casos de acidentes por contato com animais domésticos atendidos nas unidades de saúde distribuídas pelo município de Pinhais, Paraná, no período de janeiro de 2002 a dezembro de 2005. Foram utilizadas informações existentes nas unidades de saúde, contidas nas fichas de atendimento anti-rábico humano do Sistema de Informação de Agravos de Notificação (SINAN), elaboradas pelo Ministério da Saúde.

Foram considerados, para efeito de análise, os acidentes que envolveram qualquer contato que, de alguma forma, pudesse trazer algum tipo de risco ao ser humano, como mordeduras, arranhaduras e ou lambeduras. As principais informações obtidas do protocolo de estudo foram: data da notificação, idade da vítima, sexo, local do corpo acometido, tipo de ferimento, tipo de agressão, espécie do animal agressor, condição do animal, modo de ocorrência da agressão e se a ocorrência foi acidental ou pro- 
FORTES, F. S. et al.

vocada. Os dados foram tabulados em planilhas de dados e analisados segundo suas dinâmicas no referido período.

\section{RESULTADOS E DISCUSSÃO}

Durante o período de estudo foi registrado um total de 2.163 casos de acidentes com animais domésticos. O tipo de exposição de maior ocorrência foi a mordedura, com 1.972 casos $(81,9 \%)$, seguido da arranhadura com 323 casos (13,4\%). Este resultado foi semelhante ao encontrado no município de Campo Grande, MS, que revelou $81,5 \%$ de mordeduras (RIGO e HONER, 2005). Esta concentração ocorre, provavelmente, devido à conscientização da população estudada quanto ao alto risco de conta- minação pelo vírus rábico através de mordeduras, o que não ocorre nos casos de arranhaduras, de lambeduras ou pelo simples contato (GARCIA et al., 1999). Na análise dessas ocorrências, verificaram-se as principais características das pessoas expostas às agressões (TABELAS 1 a 3 ).

Quanto à distribuição por faixa etária e sexo, a maioria das vítimas pertencia à faixa de idade acima de 12 anos $(61,4 \%)$, e dentre estes uma discreta maioria do sexo masculino $(53,1 \%)$ (TABELA 1$)$. As localizações das lesões em pacientes acima de 12 anos foram mais freqüentes nas mãos e nos pés $(39,3 \%)$, seguido dos membros inferiores $(33,3 \%)$ e membros superiores $(18,7 \%)$ (TABELA 2). O acometimento de mãos e pés na maioria dos casos sugere que as vitimas interagiram com o animal no momento do acidente, quer seja pela

TABELA 1 - DISTRIBUIÇÃO ETÁRIA E POR SEXO DOS 2.163 PACIENTES EXPOSTOS ÀS AGRESSÕES POR CÃES E GATOS, ATENDIDOS NAS UNIDADES DE SAÚDE DO MUNICÍPIO DE PINHAIS, PR, NO PERÍODO DE JANEIRO DE 2002 A DEZEMBRO DE 2005.

\begin{tabular}{cccc}
\hline & $<\mathbf{1 2}$ anos 836 (38,6\%) & $>12$ anos 1.327 (61,4\%) & Total 2.163 (100\%) \\
\hline Sexo & & & \\
Masculino & $536(64,1 \%)$ & $704(53,0 \%)$ & $1.240(57,3 \%)$ \\
Feminino & $300(35,9 \%)$ & $623(47,0 \%)$ & $923(42,7 \%)$ \\
Total & $836(100 \%)$ & $1.327(100 \%)$ & $2.163(100 \%)$ \\
\hline
\end{tabular}

TABELA2 - DISTRIBUIÇÃO SEGUNDO O LOCAL DO CORPO ATINGIDO DOS 2.163 PACIENTES EXPOSTOS ÀS AGRESSÕES POR CÃES E GATOS, ATENDIDOS NAS UNIDADES DE SAÚDE DO MUNICÍPIO DE PINHAIS, PR, NO PERÍODO DE JANEIRO DE 2002 A DEZEMBRO DE 2005.

\begin{tabular}{cccc}
\hline & $<12$ anos $836(38,6 \%)$ & $>12$ anos $1.327(61,4 \%)$ & Total 2.163 (100\%) \\
\hline Local atingido & & & \\
Membros inferiores & $301(33,3 \%)$ & $545(33,3 \%)$ & $846(33,3 \%)$ \\
Membros superiores & $169(18,7 \%)$ & $306(18,7 \%)$ & $475(18,7 \%)$ \\
Cabeça ou pescoço & $185(20,5 \%)$ & $49(3,0 \%)$ & $234(9,2 \%)$ \\
Mãos ou pés & $139(15,4 \%)$ & $644(39,3 \%)$ & $783(30,8 \%)$ \\
Tronco & $78(8,6 \%)$ & $73(4,5 \%)$ & $151(6,0 \%)$ \\
Mucosas & $32(3,5 \%)$ & $20(1,2 \%)$ & $52(2,0 \%)$ \\
Total & $904(100 \%)$ & $1.637(100 \%)$ & $2.541(100 \%)$ \\
\hline
\end{tabular}

TABELA 3 - DISTRIBUIÇÃO SEGUNDO AS CARACTERÍSTICAS DO FERIMENTO DOS 2.163 PACIENTES EXPOSTOS AُS AGRESSÕES POR CÃES E GATOS, ATENDIDOS NAS UNIDADES DE SAÚDE DO MUNICÍPIO DE PINHAIS, PR, NO PERÍODO DE JANEIRO DE 2002 A DEZEMBRO DE 2005.

\begin{tabular}{cccc}
\hline & $<12$ anos $836(38,6 \%)$ & $>12$ anos $1.327(61,4 \%)$ & Total $2.163(100 \%)$ \\
\hline Ferimento & $499(59,7 \%)$ & $761(57,3 \%)$ & $1.260(58,3 \%)$ \\
Único & $328(39,2 \%)$ & $516(38,9 \%)$ & $844(39,0 \%)$ \\
Múltiplo & $9(1,1 \%)$ & $50(3,8 \%)$ & $59(2,7 \%)$ \\
Ignorado & $836(100 \%)$ & $1.327(100 \%)$ & $2.163(100 \%)$ \\
Total & & & \\
Tipo de ferimento & $338(38,5 \%)$ & $600(42,2 \%)$ & $938(40,7 \%)$ \\
Profundo & $459(52,2 \%)$ & $686(48,2 \%)$ & $1.145(49,7 \%)$ \\
Superficial & $82(9,3 \%)$ & $137(9,6 \%)$ & $220(9,6 \%)$ \\
Dilacerante & $879(100 \%)$ & $1.423(100 \%)$ & $2.303(100 \%)$ \\
Total & &
\end{tabular}


Acidentes por mordeduras de cães e gatos no município de Pinhais, Brasil de 2002 a 2005

provocação, distração ou defesa pessoal. Isso corrobora com o fato de que em 253 casos $(19,1 \%)$, a agressão foi provocada pela vítima, e auxilia na avaliação comportamental do animal, visto que uma agressão espontânea pode indicar a possibilidade de infecção pela raiva (CARAMORI JUNIOR et al., 2003, MIRANDA et al., 2003). Os ferimentos úni$\cos (57,4 \%)$ e superficiais $(48,2 \%)$ foram de maior ocorrência, também mostrando que não houve um comportamento agressivo mais severo ou contínuo destes animais (TABELA 3).

Embora os acidentes ocorridos em pacientes até 12 anos de idade tenham sido em proporção menor $(38,6 \%)$, também mostraram uma discreta tendência para o sexo masculino (58\%) (TABELA 1). A associação de indivíduos desta faixa etária ao sexo masculino pode ser explicada pela maior atividade, maior oportunidade de encontro com os animais no espaço social que ocupam, e ainda brincadeiras e atitudes bruscas que possam despertar a reação agressiva do animal (GARCIA et al., 1999). Nossos resultados condizem com outros estudos, onde as crianças, juntamente com sexo masculino e local da residência das vítimas, são considerados fatores de risco para acidentes por mordedura de cães (OZANNE-SMITH et al., 2001, LIPPOLIS et al., 2004).

Os pacientes de até 12 anos de idade mostraram um perfil diferente do local das lesões quando comparados com os adultos, com maior ocorrência nos membros inferiores $(33,3 \%)$, cabeça e pescoço $(20,5 \%)$ e membros superiores $(18,7 \%)$ (TABELA 2). A maior freqüência relativa de lesões situadas na face de crianças pode ser explicada pela menor estatura das vítimas e da forma de relacionamento com os animais (GARCIA et al., 1999). O número de casos provocados pelas próprias vítimas foi também relativamente maior nestes pacientes $(30,5 \%)$, mostrando que embora o comportamento da criança não seja intencional, algumas atividades do dia-a-dia podem parecer provocativas, irritando ou assustando os animais (DEL CIAMPO et al., 2000). O perfil da mordedura foi semelhante nas crianças, com predominância de ferimentos únicos $(59,7 \%)$ e superficiais $(52,2 \%)$ (TABELA 3).

A maioria das agressões foi causada pelos cães, com $2.074(95,9 \%)$ casos, seguido pelos gatos, com $63(2,9 \%)$ casos. Foi registrado ainda um caso com primata e um caso com morcego. O predomínio do cão como animal agressor concorda com a totalidade da literatura científica (COSTA, 1999; LOPES et al., 2001; BLATT et al., 2002; BONITO et al., 2002).

Quanto ao estado clínico dos animais agressores, $51,0 \%$ destes encontravam-se aparentemente sadios no momento do acidente. Este resultado, muito próximo ao obtido no município de Osasco, $\mathrm{SP}$, que revelou $47,8 \%$ de animais sadios (GARCIA et al., 1999), é inferior ao observado no município de Ribeirão Preto, SP (DEL CIAMPO et al., 2000). O estado clínico do restante dos animais foi desconhecido, o que resulta em uma porção considerável de animais potencialmente expostos ao risco de contrair o vírus da raiva e transmití-lo ao homem, principalmente por mordeduras. Esta constatação destaca a importância das atividades de educação em saúde visando conscientizar os proprietários de animais de estimação da necessidade de realizar a vacinação anti-rábica anualmente, reduzindo os riscos para toda a comunidade.

Apesar do conhecimento da procedência do animal, seu relacionamento com a vítima e o local onde a agressão ocorreu serem fundamentais para o estudo epidemiológico e prevenção dos acidentes, estas informações não constam da ficha de atendimento anti-rábico do SINAN. Deste modo, embora outros estudos tenham mostrado que a maioria das vítimas reconhece os respectivos cães agressores (MENDEZ et al., 2002; MITCHELL et al., 2003; LIPPOLIS et al., 2004), no presente estudo a ficha do SINAN não permitiu esta inferência com segurança, apontando apenas que a maioria dos agravos ocorreu em horas de lazer (47,8\%). Embora outros estudos tenham mostrado que o cão agressor habita a casa das vítimas ou propriedades vizinhas (DEL CIAMPO et al., 2000; BEAVER, 2001; MIRANDA et al., 2003;), a ficha atual do SINAN também não permitiu afirmar se houve mesmo este perfil epidemiológico no presente estudo. Assim sendo, embora a ficha de atendimento anti-rábico do SINAN tenha sido um marco na notificação de mordeduras e outros agravos, é de suma importância que sejam incluídos em seu formulário duas questões: "quem é o dono do animal? 1.vítima ou 2.familiar da casa, 3.vizinho, 4.conhecido, 5.desconhecido, 6.outros"; "onde ocorreu o acidente? 1.dentro da casa da vítima, 2. de vizinhos, 3. familiares, 4 . outra casa, 5 . na rua, 6.outros". Com estas informações adicionais, não apenas o município de Pinhais, mas todos os municípios que utilizam estas fichas, poderiam instituir um programa de prevenção de acidentes por mordeduras focado no perfil do cão agressor, da vítima agredida, e do local da agressão. Em outros estudos também se observou a falta de informações na ficha de investigação (RIGO e HONER, 2005, MUNDIM et al., 2007).

Os meses de maior notificação de mordeduras variaram no decorrer do período e incluindo os meses de férias escolares, o que corrobora parcialmente com o já observado em outros estudos (FRANGAKIS e PETRIDOU, 2003). No entanto, houve uma menor ocorrência de agressões nos meses de março, abril e maio, sem uma aparente explicação para o observado. 
FORTES, F. S. et al.

\section{CONCLUSÕES}

O presente estudo mostrou que apesar do Sistema de Informação de Agravos de Notificação (SINAN) ser a principal fonte de investigação de casos de atendimento anti-rábico e acidentes por mordeduras, a ficha epidemiológica não contém dados que especifiquem a situação de domicílio do animal agressor, sua relação com a vítima e o espaço geográfico onde a agressão ocorreu, sendo essas informações fundamentais para delinear 0 perfil do animal agressor e da vítima, e conseqüentes programas de prevenção destes acidentes e de educação em saúde.

Visto que não houve, durante o período analisado, nenhuma redução dos acidentes, quer quantitativa ou qualitativamente, faz-se necessário desenvolver um trabalho educativo junto à população para conscientizá-la sobre os riscos e a gravidade de agressões ocasionadas por animais, destacando-se que a prevenção é possível de ser realizada, quando se conhecem os fatores envolvidos na gênese dos acidentes. A análise das fichas poderá ser utilizada como instrumento de monitoramento da eficácia destes programas, norteando a consolidação dos mesmos.

\section{REFERÊNCIAS}

ACHA, P.N.; SZYFRES, B. Zoonoses and communicable diseases common to man and animals. Washington: Pan American Health Organization, 2003, p.246-275.

ARAÚJO, F.A.A. Situação epidemiológica da raiva: panorama brasileiro. In: SIMPÓSIO INTERNACIONAL PROGRAMA DE TREINAMENTO "CONTROLE DE ZOONOSES E AS INTERAÇÕES HOMEM-ANIMAL”, 2001, Embu (SP). Anais.: São Paulo, 2001.

BEAVER, B.V. Comportamento canino: Um guia para veterinários. São Paulo: Roca, 2001, p.189249.

BLATT, M.C.S.; MUNDIM, A.P.M.; ROCHA, S.M.; SINKOC, A.L.; SANTOS, J.C.Q. Caracterização das agressões por cães e gatos no município de Cuiabá no período de janeiro/2000 a outubro/2001. In: CONGRESSO BRASILEIRO DE MEDICINA VETERINÁRIA, XXIX. 2002, Gramado (RS), Anais... Gramado (RS), 2002.

BONITO, R.F.; OLIVEIRA, N.M.; NISHIOKA, S.A.
Aspectos epidemiológicos das agressões animais. Revista Brasileira de Epidemiologia - Livro de Resumos, Curitiba (PR), v.5 Supl 1, p.262, 2002.

CARAMORI JUNIOR, J.G.; LUBAS, M.A.; KAWATAKE, M.S. Inquérito Epidemiológico sobre características da população canina e felina de um bairro próximo à zona rural em Cuiabá-MT, visando o controle da raiva animal. Revista da Sociedade Brasileira de Medicina Tropical, v.36, p.419-420, 2003.

CHANG, Y.; MCMAHON, J.E.; HENNON, D.L.; LAPORTE, R.E.; COBEN, J.H. Dog bite incidence in the city of Pittsburgh: a capture-recapture approach. American Journal of Public Health, v.87, p.1703-5, 1997.

COSTA, W.A. Aspectos Práticos na Prevenção da Raiva Humana. Jornal de Pediatria, v.75, n.1, p.135-148, 1999.

DEL CIAMPO, L.A.; RICCO, R.G.; ALMEIDA, C.A.N. de; BONILHA, L.R. de C.M.; SANTOS, T.C.C. dos. Acidentes de Mordeduras de cães na infância. Revista de Saúde Pública, v.34, n.4, p.411-412, 2000.

ETTINGER, S.J.; FELDMAN, E.C. Tratado de Medicina Interna Veterinária, 1997. v.1, p.242-252.

FENNER, F.; BACHANN, P.A.; GIBBS, E.P.J.; MURPHI, F.A.; STUDDERT, M.J.; WHITE, D.O. Veterinary virology, 2.ed. New York: Academic Press, 1993.

FRANGAKIS, C.E. e PETRIDOU, E. Modelling risk factors for injuries from dog bites in Greece: a case only design and analysis. Accidents Analysis and Prevention, v.35, n.3, p.435-438, 2003.

GARCIA, R.C.M.; VASCONCELLOS, S.A.; SAKAMOTO, S.M.; LOPEZ, A.C. Análise de tratamento anti-rábico humano pós-exposição em região da Grande São Paulo, Brasil. Revista de Saúde Pública, v.33, n.3, p.295-301, 1999.

HUGH-JONES, M.E.; HUBBERT, W.T.; HAGSTAD, $H . V$. Zoonoses: recognition, control and prevention. 1ed., Ames, lowa State University Press, 1995. $369 \mathrm{p}$.

LIPPOLIS, M.; DUTRA SOBRINHO, J.P.; BENITES, N.R. Epidemiologia e avaliação dos fatores de risco associados a acidentes por mordedura de cães em humanos, no Município de Guarulhos, Estado de São 
Acidentes por mordeduras de cães e gatos no município de Pinhais, Brasil de 2002 a 2005

Paulo, de 1997-2003. Arquivo Instituto Biológico, v.71, (supl.), p.1-749, 2004.

LOPES, C.A.P.; FRANCO, S.O.; NUNES-PINHEIRO, D.C.S.; LEITE, A.K.R.M. Profilaxia anti-rábica pósexposição para agressões por animais errantes no município de Fortaleza, Ceará. Revista Ciência Animal, v.11, n.1, p.82-84, 2001.

MENDEZ, G.R.; GOMES, T.M.; SOMOZA, A.I.; LIRAS, M.J.; PAIS, P.E.; VELA, N.D. Dog bite-related injuries treated in a pediatric surgery department: analysis of 654 cases in 10 years. Anales Españoles de Pediatría, v.56, n.5, p.425-429, 2002.

MINISTÉRIO DA SAÚDE, Coordenação de controle de zoonoses e Animais peçonhentos. Manual de Normas Técnicas para Profilaxia de Raiva em Humanos. Brasília (DF); 2002.

MINISTÉRIO DA SAÚDE. Sistema nacional de vigilância em saúde: Relatório de situação: Paraná, Secretaria de Vigilância em Saúde - 2.ed. - Brasília: Ministério da Saúde, 2006. 24 p.: il. color. - (Série C. Projetos, Programas e Relatórios).

MIRANDA, C.F.J.; SILVA, J.A.; MOREIRA, E.C. Raiva Humana transmitida por cães: áreas de risco em Minas Gerais, Brasil, 1991-1999. Cadernos de Saúde Pública, v.19, n.1, p.91-99, 2003.

MITCHELL, R.B.; NANEZ, G.; WAGNER, J.D.; KELLY, J. Dog bites of the scalp, face, and neck in children. Laryngoscope, v.113, n.3, p.492-495, 2003.

MUNDIM, A.P.M. Exposição à raiva humana no município de Cuiabá-MT: Epidemiologia e avaliação das medidas preventivas. 2005. 108p. Dissertação (Mestrado em Saúde Coletiva)-Universidade Federal do Mato Grosso, Cuiabá.

MUNDIM, A.P.M.; SCATENA, J.H.G.; FERNANDES, C.G.N. Agressividade canina a seres humanos: reação normal ou alteração comportamental motivada pela raiva? Clínica Veterinária, n.67, p.84-88, 2007.

OVERALL, K.L.; LOVE, M. Dog bites to humans demography, epidemiology, injury, and risk. Journal of the American Veterinary Medical Association, v.218, n.12, p.1923-1934, 2001.

OZANNE-SMITH, J.; ASHBY, K.; STATHAKIS, V.Z.
Dog bite and injury prevention-analysis, critical review, and research agenda. Injury Prevention, v.7, n. 4 , p. 321-326, 2001.

PLAUT, M.; ZIMMERMAM, E. M.; GOLDSTEIN, R. A. Health hazards to humans associated with domestic pets. Annual Review in Public Health, v.17, p.221245, 1996.

RIGO, L.; HONER, M.R. Análise da profilaxia da raiva humana em Campo Grande, Mato Grosso do Sul, Brasil, em 2002. Cadernos de Saúde Pública, v.21, n.6, p.1939-1945, 2005.

SCHABBACH, C.H. Agressões por cães em Rio Grande - RS: Um estudo da classificação por situação de domicílio. Porto Alegre-RS; 2004. [Monografia Especialização - Departamento de Medicina Social da Universidade Federal do Rio Grande do Sul].

SCHALAMON, J.; AINOEDHOFER, H.; SINGER, G.; PETNEHAZY, T.; MAYR, J.; KISS, K.; HÖLLWARTH, M.E. Analysis of Dog Bites in Children Who Are Younger Than 17 Years. Pediatrics, v.117, n.3, 374-379, 2006.

SCHOENDORFER, L.M.P. Interação HomemAnimal de Estimação na cidade de São Paulo - Manejo Inadequado e as Conseqüências em Saúde Pública. São Paulo, 2001. [Dissertação de Mestrado - Faculdade de Saúde Pública da Universidade de São Paulo].

TAN, R.L.; POWELL, K.E.; LINDEMER, K.M.; CLAY, M.M.; DAVIDSON, S.C. Sensitivities of three county health department surveillance systems for childrelated dog bites: 261 cases (2000). Journal of the American Veterinary Medical Association, v.225, n.11, p.1680-1683, 2004.

Recebido para publicação: $21 / 05 / 2007$ Aprovado: 26/09/2007 